\title{
ON MEROMORPHIC SOLUTIONS OF ALGEBRAIC DIFFERENTIAL EQUATIONS
}

\author{
BY
}

SH. STRELITZ

\begin{abstract}
The Malmquist Theorem is generalized for equations of the type $R\left(z, w, w^{\prime}, \ldots, w^{(n)}\right)=P(z, w) / Q(z, w)$ where $P, Q$ and $R$ are polynomials of $w$ and $w, w^{\prime}, \ldots, w^{(n)}$ respectively with meromorphic coefficients of finite order.
\end{abstract}

1. In this paper we consider the differential equation

$$
R\left(z, w, w^{\prime}, \ldots, w^{(n)}\right)=P(z, w) / Q(z, w)
$$

where $P, Q$ and $R$ are polynomials of $w$ and $w, w^{\prime}, \ldots, w^{(n)}$ respectively with meromorphic coefficients of $z$ in the plane $|z|<\infty$.

We generalized in [4] the well-known Malmquist Theorem [2] for equations of type (1.1), where $P, Q$ and $R$ were polynomials of all their corresponding variables and obtained there under these conditions that if (1.1) has a transcendental meromorphic solution in $|z|<\infty$ then $Q(z, w)$ does not depend on $w$. In this paper we generalize the indicated theorem for (1.1) described above.

In order to formulate the main assertion of this paper we rewrite $P, Q$ and $R$ of (1.1) in the following forms:

$$
\begin{aligned}
R\left(z, w, w^{\prime}, \ldots, w^{(n)}\right) & =\sum_{i_{0}+i_{j}+\cdots+i_{n}=0}^{m} R_{i_{0} i_{s} \cdots i_{n}}(z) w^{i_{0}}\left(w^{\prime}\right)^{i_{3}} \cdots\left(w^{(n)}\right)^{i_{n}} \\
P(z, w) & =\sum_{k=0}^{p} P_{k}(z) w^{p-k} \\
Q(z, w) & =\sum_{j=0}^{q} Q_{j}(z) w^{p-k}
\end{aligned}
$$

We will use the following notations.

(1) We denote by $\rho(f)$ the order of the meromorphic function $f$ and put

$$
\lambda=\max \left\{\rho\left(R_{i_{0} i_{1} \cdots i_{n}}\right), \rho\left(P_{k}\right), \rho\left(Q_{j}\right)\right\}
$$

where the maximum is taken over all the possible values of the indices $i_{s}, k$ and $j$.

(2) We denote the set of all the polynomials

$$
T(z, w)=\sum_{s=0}^{t} T_{s}(z) w^{t-s}
$$

where all the $T_{s}(z)$ are meromorphic functions in $|z|<\infty$ by $\mathfrak{W}$.

Received by the editors May 7, 1979.

AMS (MOS) subject classifications (1970). Primary 34A20; Secondary 30A70.

Key words and phrases. Meromorphic functions, algebraic differential equations. 
Definition 1. The polynomial $T_{0}(z, w) \in \mathfrak{M}$ which depends explicitly on $w$ is called a nontrivial divisor of $T(z, w)$ in $\mathfrak{M}$ if there is a third nontrivial $T_{1} \in \mathfrak{M}$ (that is, $T_{1}(z, w)$ depends explicitly on $\left.w\right)$ such that $T \equiv T_{1} T_{0}$.

Definition 2. Two polynomials $T_{1}, T_{2} \in \mathfrak{M}$ are called mutually prime if they have no common nontrivial divisors.

We are now able to formulate the main result of this paper.

TheOREM. Consider (1.1). Let $P(z, w)$ and $Q(z, w)$ be mutually prime in $\mathfrak{M}$ and let $\lambda<\infty$. If (1.1) has a transcendental meromorphic solution $w(z)$ in $|z|<\infty$ of order $\rho>\lambda$ then $Q(z, w)$ does not depend on $w$.

REMARK. In this theorem it is impossible in general to put $\rho(w)>\lambda$. Indeed let $p_{k}(z)$ and $q_{j}(z)$ be transcendental meromorphic functions with $\rho\left(p_{k}\right)<\rho, \rho\left(q_{j}\right)<\rho$ (for all $k$ and $j$ ) and $f(z)$ a meromorphic function of order $\rho$. Then the equation

$$
w^{\prime \prime}=f^{\prime \prime} \cdot \frac{\sum_{k=0}^{n} q_{k}(z) f^{n-k}}{\sum_{j=0}^{m} p_{j}(z) f^{m-j}} \cdot \frac{\sum_{j=0}^{m} p_{j}(z) w^{m-j}}{\sum_{k=0}^{n} q_{j}(z) w^{n-j}}
$$

has a solution $f(z)$ of order $\rho$.

In the following sections of this paper we prove the theorem formulated above.

2. We can always assume that the degree, $d(P)$, of $P(z, w)$ in respect to $w$ is less than the degree, $d(Q)$, of $Q(z, w)$ in respect to $w$; $d(P)\langle d(Q)$. Indeed if $d(P)\rangle$ $d(Q)$ then dividing $P(z, w)$ by $Q(z, w)$ as polynomials of $w$, we will separate the entire polynomial part (in respect to $w$ ), $s(z, w)$, and will obtain a remainder, $P_{1}(z, w) / Q(z, w)$, with a degree, $d\left(P_{1}\right)$, of $P_{1}(z, w)$ lower than the degree, $d(Q)$, of $Q(z, w)$ :

$$
P(z, w) / Q(z, w)=S(z, w)+P_{1}(z, w) / Q(z, w)
$$

with $d\left(P_{1}\right)<d(Q)$. From (1.1) now follows $R\left(z, w, w^{\prime}, \ldots, w^{(n)}\right)-S(z, w)=$ $P_{1}(z, w) / Q(z, w)$. Denoting

$$
R\left(z, w, w^{\prime}, \ldots, w^{(n)}\right)-S(z, w)=R_{1}\left(z, w, w^{\prime}, \ldots, w^{(n)}\right)
$$

we get a new equation

$$
R_{1}\left(z, w, w^{\prime}, \ldots, w^{(n)}\right)=P_{1}(z, w) / Q(z, w)
$$

where $P_{1}(z, w) / Q(z, w)$ has the required property. We assume that already in (1.1) the degree of $P(z, w)$ is lower than the degree of $Q(z, w)$ in respect of $w$.

In order to prove our theorem we suppose that $Q(z, w)$ depends explicitly on $w$. Let $w(z)$ be a meromorphic solution of order $\rho>\lambda$ of (1.1). We substitute in (1.1) $w$ for $w(z)$. By a suitable transformation

$$
w=(\beta u+1) /(u+1)
$$

with an appropriately chosen $\beta,(1.1)$ will be reduced to

$$
R^{*}\left(z, u, u^{\prime}, \ldots, u^{(n)}\right)=P^{*}(z, u) / Q^{*}(z, u)
$$

with the following properties: 
(i) $P^{*}(z, u), Q^{*}(z, u)$ and $R^{*}\left(z, u, u^{\prime}, \ldots, u^{(n)}\right)$ are polynomials of $u$ and $u, u^{\prime}, \ldots, u^{(n)}$ respectively with meromorphic coefficients of order no more than $\lambda$;

(ii) $P^{*}(z, w)$ and $Q^{*}(z, w)$ are mutually prime in $\mathfrak{M}$;

(iii) the function $Q^{*}(z, u(z))$, where $u(z)$ is the meromorphic solution of (2.2) corresponding to $w(z)$ (of order $\rho: \rho>\lambda$ ), does not vanish at the poles of the function $u(z)$ and

(iv) $a=\infty$ is not a deficiency value of $u(z)$.

In order to prove these properties consider now the zeros of the function $Q^{*}(z, u(z))$. In the poles of $u(z)$ according to (2.1)

$$
\left.w(z)\right|_{u=\infty}=\beta \text {. }
$$

Further,

$$
\begin{aligned}
Q\left(z, \frac{\beta u+1}{u+1}\right) & =\sum_{j=0}^{q} Q_{j}(z)\left(\frac{\beta u+1}{u+1}\right)^{q-j} \\
& =\frac{1}{(u+1)^{q}} \sum_{j=0}^{q} Q_{j}(z)(\beta u+1)^{q-j}(u+1)^{j}=\frac{Q^{*}(z, u)}{(1+u)^{q}}
\end{aligned}
$$

and

$$
\frac{P(z, w)}{Q(z, w)}=\frac{P\left(z, \frac{\beta u+1}{u+1}\right)(1+u)^{q}}{Q^{*}(z, u)}=\frac{P^{*}(z, u)}{Q^{*}(z, u)}
$$

with the polynomial

$$
P^{*}(z, u)=P\left(z, \frac{\beta u+1}{u+1}\right)(1+u)^{q}
$$

We have

$$
\left.Q\left(z, \frac{\beta u+1}{u+1}\right)\right|_{u=\infty}=Q(z, \beta) .
$$

Let $\left\{z_{n}\right\}$ be the sequence of all the zeros of the function $Q(z, w(z)): Q\left(z_{n}, w\left(z_{n}\right)\right)=$ $0, n=1,2,3, \ldots$ We now point out a number $\beta$ such that

$$
w\left(z_{n}\right) \neq \beta, \quad n=1,2,3, \ldots
$$

Suppose $\left\{z_{p}^{\prime}\right\}$ is the sequence of all the solutions of the equation $w(z)=\beta$ :

$$
w\left(z_{j}^{\prime}\right)=\beta, \quad j=1,2,3, \ldots
$$

Obviously $z_{j}^{\prime} \neq z_{k} ; j, k=1,2,3, \ldots$ At the point $z_{j}^{\prime}$, in view of their construction,

$$
Q\left(z_{j}^{\prime}, w\left(z_{j}^{\prime}\right)\right) \neq 0, \quad j=1,2,3, \ldots
$$

So that $Q(z,(\beta u+1) /(u+1))$ does not vanish in the poles of $u$. From (2.4) we now infer that all the solutions of $Q^{*}(z, u)=(u+1)^{q} Q(z,(\beta u+1) /(u+1))=0$ are different from the poles of $u(z)$. Besides $\beta$ can be chosen in such a manner that $Q^{*}(z, u)$ will depend explicitly on $u$ and additionally so that $a=\infty$ will be a nondeficiency value of $u(z)$ since the deficiency values form only a countable set. It is obvious also that if in (1.1) $P$ and $Q$ are mutually prime in $\mathbb{M}$, then $P^{*}(z, u)$ and $Q^{*}(z, u)$ will be mutually prime in $\mathfrak{M}$ too.

Thus without restricting the generality we can assume that already in (1.1) 
$Q(z, w(z)) \neq 0$ at the poles of $w(z)$ and that $a=\infty$ is not a deficiency value of $w(z)$.

3. It follows now from (1.1) under our assumptions of the $\$ 2$ that $P(z, w(z))$ vanishes at every point where $Q(z, w(z))$ vanishes with the exception maybe of a sequence of poles of the function $R_{i_{0} i_{1} \cdots i_{n}}(z)$ (see (1.2)) whose convergence exponent is not greater than $\lambda$. Indeed, at a point $z_{0}$ where $Q\left(z_{0}, w\left(z_{0}\right)\right)=0$, according to our construction $w\left(z_{0}\right) \neq \infty$, so that if $P\left(z_{0}, w\left(z_{0}\right)\right) \neq 0$ then it is indispensable that

$R_{0}\left(z_{0}\right)=R\left(z_{0}, w\left(z_{0}\right), \ldots, w^{(n)}\left(z_{0}\right)\right)=\infty, \quad R_{0}(z) \equiv R\left(z, w(z), \ldots, w^{(n)}(z)\right)$

i.e., the function $R_{0}(z)$ will have a pole in $z_{0}$. But this is possible only if $z_{0}$ is a pole of at least one of the coefficients $R_{i_{0} i_{1} \cdots i_{n}}(z)$ in (1.2). Since $w\left(z_{0}\right) \neq \infty$ the multiplicity of the pole of $R_{0}(z)$ cannot be greater than the multiplicity of the pole of the corresponding functions $R_{i_{0} i_{1} \cdots i_{n}}(z)$. But the last functions are of order not more than $\lambda$, so that the convergence exponent of the poles of $R_{0}(z)$ is not exceeding $\lambda$.

Consider now the common zeros of $P(z, w)$ and $Q(z, w)$, that is the set of all the solutions of the system of equations

$$
P(z, w)=0, \quad Q(z, w)=0 .
$$

The resultant $D(z)$ of this system is a meromorphic function of order no greater than $\lambda$ and cannot vanish identically because $P(z, w)$ and $Q(z, w)$ are mutually prime in $\mathfrak{M}$ according to our conditions of the theorem. Consequently the sequence of the zeros of the function $Q(z, w(z))$ has a convergence exponent not greater than $\lambda$. Thus there is a representation

$$
Q(z, w(z)) \equiv f(z) / F(z),
$$

where $f(z)$ is an entire function of order not greater than $\lambda, F(z)$ is another entire function.

LEMMA 1. $F(z)$ in the representation (3.2) is an entire function of order $\rho$.

RemarK. We restrict ourself with $\rho<\infty$. The case $\rho=\infty$ can be dealt with literally in the same way.

Proof of Lemma 1. We consider the function

$$
Q(z, w(z)) \equiv \sum_{j=0}^{q} Q_{j}(z) w^{q-j}(z) .
$$

Since $a=\infty$ is not a deficiency value of $w(z)$ (see property (iv) in $\S 2$ ) then the convergence exponent of the sequence of the poles of (3.3) equals $\rho$ and $Q(z, w(z))$ is of order $\rho$. Indeed

As we saw in $\S 2$

$$
\sum_{j=0}^{q} Q_{j}(z) w^{q-j}(z)=w^{q}(z)\left[Q_{0}(z)-\sum_{j=1}^{q} Q_{j}(z) / w^{j}(z)\right] .
$$

$$
Q_{0}(z)-\sum_{j=1}^{q} Q_{j}(z) / w^{j}(z) \neq 0
$$


at the poles of $w(z)$ so that $Q(z, w(z))$ is of order not less than $\rho$, because the convergence exponent of its poles as it follows from (3.4) and (3.5) is not less than $\rho$. On the other hand, $Q(z, w(z))$ is at most of order $\rho$. Thus the order of $Q(z, w(z))$ equals $\rho$.

Equality (3.2) now shows that $F(z)$ is an entire function of order $\rho$ since $Q(z, w(z))$ is of order $\rho$ and $f(z)$ of order $\lambda<\rho$. Lemma 1 is proven.

4. We continue the proof of the theorem.

Let $\zeta$ be a maximal point of the function $|F(z)|$ on the circle $|z|=r$, that is,

$$
|F(\zeta)|=\max _{|r|=r}|F(z)|=M(r, F), \quad|\zeta|=r
$$

Denote

$$
K\left(r_{0}, F\right)=\lim _{r \rightarrow r_{0}+0} \frac{r M^{\prime}(r, F)}{M(r, F)} .
$$

It is known [3, p. 148] that for each $\zeta,|\zeta|=r$ with the exception of a sequence of intervals $\bar{I}$ on the $r$-axis with bounded logarithmic measure (that is $\int_{\bar{I}}(d r / r)<\infty$ ).

$$
F\left(\zeta e^{\eta}\right)=(1+o(1)) F(\zeta), \quad\left|F\left(\zeta e^{\eta}\right)\right|=(1+o(1)) M(r, F), \quad r \notin \bar{I},
$$

for

$$
|\eta| \leqslant 1 / K \ln ^{1+\alpha} K, \quad K=K(r, F),
$$

with an arbitrary fixed $\alpha>0, \bar{I}=\bar{I}(\alpha)$. For such $\eta$

$$
|\zeta|(1-2|\eta|)<\left|\zeta e^{\eta}\right|<|\zeta|(1+2|\eta|)
$$

because $K(r, F) \rightarrow \infty$ (see [3, p. 66]). Hence according to equation (4.3), $|F(z)|=$ $(1+o(1)) M(r, F)$ in the circle $C_{\zeta}$ :

$$
|z-\zeta| \leqslant|\zeta||\eta| / 2
$$

( $\eta$ is given by (4.4)).

Denote $H=\cup_{\zeta} C_{\zeta},|\zeta| \notin \bar{I}$. We will now construct a sequence $I_{1}=$ $\cup_{j}\left(R_{j}, R_{j}^{\prime}\right)$ on the $r$-axis such that $\left(R_{i}, R_{i}^{\prime}\right) \cap\left(R_{j}, R_{j}^{\prime}\right)=\varnothing, i \neq j, I_{1} \cap \bar{I}=\varnothing$, mes $I_{1}=\infty$ and

$$
\lim _{\substack{r \rightarrow \infty \\ r \in I_{1}}} \frac{\ln \ln M(r, F)}{\ln r}=\rho .
$$

In order to prove it we note that there is a sequence $I_{0}$ of intervals $I_{0}=$ $\cup_{i=1}^{\infty}\left(R_{j}, R_{j}^{\prime}\right),\left(R_{j}, R_{j}^{\prime}\right) \cap\left(R_{i}, R_{i}^{\prime}\right)=\varnothing, i \neq j$, mes $I_{0}=\infty$ such that

$$
\lim _{\substack{r \rightarrow \infty \\ r \in I_{0}}} \frac{\ln \ln M(r, F)}{\ln r}=\rho .
$$

This is obvious if the lower and upper orders of $F(z)$ coincide. To prove (4.8) suppose now that

$$
\lim _{r \rightarrow \infty} \frac{\ln \ln M(r, F)}{\ln r}<\varlimsup_{r \rightarrow \infty} \frac{\ln \ln M(r)}{\ln r}=\rho .
$$

In this case there is an infinite sequence of local maximal points $R_{j} \uparrow \infty$ such that 


$$
\lim _{j \rightarrow \infty} \frac{\ln \ln M\left(R_{j}, F\right)}{\ln R_{j}}=\rho .
$$

Let $N>1$ be an arbitrary fixed number and $R_{j}^{*}$ an arbitrary point on the segment $\left[R_{j}, N R_{j}\right], j=1,2,3, \ldots$ Evidently

$$
\frac{\ln \ln M\left(R_{j}^{*}, F\right)}{\ln R_{j}^{*}} \geqslant \frac{\ln \ln M\left(R_{j}, F\right)}{\ln R_{j}} \cdot \frac{\ln R_{j}}{\ln N R_{j}} \underset{j \rightarrow \infty}{\rightarrow} \rho .
$$

We assume $I_{0}=\cup_{j=1}^{\infty}\left(R_{j}, N R_{j}\right)$. Obviously the sequence $\left\{R_{j}\right\}$ can be chosen so rare that $\left(R_{j}, N R_{j}\right) \cap\left(R_{i}, N R_{i}\right)=\varnothing, i \neq j$. Define $I_{1}=I_{0} \backslash I$. We will show now that mes $I_{1}=\infty$. Indeed

$$
\int_{I_{1}} \frac{d r}{r}=\int_{I_{0} \backslash \bar{I}} \frac{d r}{r}=\sum_{j=1}^{\infty} \int_{R_{j}}^{N R_{j}} \frac{d r}{r}-\int_{\bar{I}} \frac{d r}{r}=\sum_{j=1}^{\infty} \ln N-\int_{\bar{I}} \frac{d r}{r}=\infty
$$

since $\int_{\bar{I}}(d r / r)<\infty$ (see above in this section). Thus

$$
\infty=\int_{I_{1} \cap\{r>1\}} \frac{d r}{r}<\int_{I_{1}} d r=\operatorname{mes} I_{1} .
$$

We return now to the representation (3.2). The function $f(z)$ there is of order $\lambda$, so that for a given $\varepsilon>0$

$$
|f(z)|<\exp r^{\lambda+\varepsilon} .
$$

On the other hand for $\zeta \in H, r=|\zeta| \in I_{1}$ and a given $\varepsilon>0$ according to (4.3)

$$
|F(\zeta)|=(1+o(1)) M(r, P)>\exp r^{p-\varepsilon} .
$$

From (4.9) and (4.10) now follows: for $\zeta \in H,|\zeta|=r \in I_{1}$

$$
|Q(\zeta, w(\zeta))|=\left|\frac{f(\zeta)}{F(\zeta)}\right|<\frac{\exp r^{\lambda+\varepsilon}}{\exp r^{\rho-\varepsilon}}=e^{-(1+o(1)) r^{p-\varepsilon}} .
$$

5. Lemma 2. For each $\omega>0$ with $\rho>\lambda+\omega$ there is a sequence of intervals $I$ on the r-axis with mes $I=\infty$ such that for all $\zeta \in H,|\zeta| \in I$

$$
\left|w^{(\nu)}(\zeta)\right|<r^{\nu(\rho+1)} e^{(2 r)^{\rho-\omega}}, \quad \nu=0,1,2,3, \ldots, N_{0},
$$

where $N_{0}$ is an arbitrary positive integer and $I=I\left(N_{0}\right)$.

Proof. Let $h(z)$ be an entire function of order $\mu$. It is known (see for example [1, p. 22]) that outside a set $H_{0}$ of circles $E_{k}$ with union $I^{*}$ of their radii $I_{k}^{*}$ : $\cup_{k=1}^{\infty} I_{k}^{*}=I^{*}$ of finite measure: mes $I^{*}<\infty$

$$
e^{-r^{\mu+\varepsilon}}<|h(z)|<e^{r^{++e}}
$$

for an arbitrary $\varepsilon>0$ and $r>r_{0}(\varepsilon)\left(r \notin I^{*}\right)$. Each function $Q_{k}(z)$ (see (1.2)) is a meromorphic function of order not more than $\lambda$ and can be represented as a fraction of two entire functions of the same order as $Q_{k}(z): Q_{k}(z)=h_{1}(z) / h_{2}(z)$. As above, there is a set $H_{0}$ of circles with union of radii $I^{*}$ of finite measure, mes $I^{*}<\infty$, such that for $z \notin H_{0}$ the functors $h_{1}(z)$ and $h_{2}(z)$ satisfy inequality (5.2'). Then for $z \notin H_{0}$

$$
e^{-(2 r)^{\lambda+\varepsilon}}<\left|Q_{k}(z)\right|<e^{(2 r)^{\lambda+\varepsilon},}
$$

for $r>r_{0}(\varepsilon)$. 
Denote $I=I_{1} \backslash I^{*}=I_{1} \backslash \bigcup_{k=1}^{\infty} I_{k}^{*}$ (the definition of $I_{1}$ is given in the previous section). Suppose now that (5.1) is already wrong for $\nu=0$. Then there is a certain $\omega_{0}>0$ with $\rho>\lambda+\omega_{0}$ and a sequence $\left\{\zeta_{j}\right\},\left\{\zeta_{j}\right\} \in I$ such that

$$
\left|w\left(\zeta_{j}\right)\right| \geqslant \exp r_{j}^{\rho-\omega_{0}}, \quad r_{j}=\left|\zeta_{j}\right| \in I, \quad r_{j} \uparrow \infty .
$$

We have

$$
\begin{aligned}
|Q(\zeta, w(\zeta))| & =\left|\sum_{k=0}^{q} Q_{k}(\zeta) w^{q-k}(\zeta)\right|>\left|Q_{0}(\zeta) w^{q}(\zeta)\right|-\sum_{k=1}^{q}\left|Q_{k}(\zeta)\right|\left|w^{q-k}(\zeta)\right| \\
& =\left|w^{q}(\zeta)\right|\left\{\left|Q_{0}(\zeta)\right|-\sum_{k=1}^{q}\left|Q_{k}(\zeta)\right||w(\zeta)|^{-k}\right\},
\end{aligned}
$$

whence in view of (4.3) and (4.2),

$$
\begin{aligned}
\left|Q\left(\zeta_{j}, w\left(\zeta_{j}\right)\right)\right| & \geqslant \exp q r_{j}^{\rho-\omega_{0}}\left\{\exp -r_{j}^{\lambda+e}-\exp r_{j}^{\lambda+e} \sum_{k=1}^{q} \exp -k r_{j}^{\rho-\omega_{0}}\right\} \\
& \geqslant \exp q r_{j}^{\rho-\omega_{0}}\left\{\exp -r_{j}^{\lambda+e}-q \exp \left(-r_{j}^{\rho+\omega_{0}}+r_{j}^{\lambda+e}\right)\right\} \\
& =\exp \left((1+o(1)) q r_{j}^{\rho+\omega_{0}}\right)
\end{aligned}
$$

for large enough $j$ and sufficiently small $\varepsilon$ (since $\rho>\lambda+\omega_{0}$ ). The last inequality contradicts (4.11). Thus for each $\omega>0$ with $\rho-\omega>\lambda$

$$
|w(\zeta)|<e^{r e-\omega}, \quad r=|\zeta| \in I, \quad r>r_{0}(\omega) .
$$

Now let $\zeta \in H$ with $|\zeta| \in I$. Then

$$
w^{(v)}(\zeta)=\frac{\nu !}{2 \pi i} \int_{C(u-\zeta)^{\nu+1}} \frac{w(u)}{(u)} \quad C:|u-\zeta|=\frac{|\zeta||\eta|}{2},
$$

where according to (3.4) we can choose

$$
|\eta|=\left(K \ln ^{l+\alpha} K\right)^{-1}, \quad K=K(r, F) .
$$

We obtain for $|\xi|=r \in I$

$$
\begin{aligned}
\left|w^{(\nu)}(\zeta)\right| & <\frac{2^{\nu} \cdot \nu !}{|\zeta|^{\nu}|\eta|^{\nu}} \max _{|u|<|\zeta|(1+|\eta| / 2)}|w(u)| \\
& =\frac{2^{\nu}}{r^{\nu}}\left(K \ln ^{1+\alpha} K\right)^{\nu} M\left(r+\frac{r|\eta|}{2}, w\right), \quad K=K(r, F),
\end{aligned}
$$

and in respect to (5.4) and (5.5) for $\zeta \in H,|\zeta| \in I$

$$
\left|w^{(\nu)}(\zeta)\right|<\frac{2^{\nu} \cdot \nu !}{|\zeta|^{\nu}}\left(K \ln ^{1+\alpha} K\right)^{\nu} \exp \left[r+\frac{r}{2}\left(K \ln ^{1+\alpha} K\right)^{-1}\right]^{\rho-\omega} .
$$

It is known $([3, \mathrm{p} .35])$, that

$$
\varlimsup_{r \rightarrow \infty} \frac{\ln K(r, F)}{\ln r}=\varlimsup_{r \rightarrow \infty} \frac{\ln \ln M(r, F)}{\ln r}=\rho .
$$

Thus for a given $\varepsilon_{0}: 1>\varepsilon_{0}>0$ and $r>r_{0}\left(\varepsilon_{0}\right)$

$$
K(r, F)<r^{p+e_{0}} \text {. }
$$

From (5.6) and (5.7) we obtain 
$\left|w^{(\nu)}(\zeta)\right|<\nu !\left(\frac{2}{r}\right)^{\nu}\left(r^{\rho+\varepsilon_{0}} \ln ^{1+\alpha} r^{\rho+\varepsilon_{0}}\right)^{\nu} \exp (2 r)^{\rho-\omega}<r^{\nu(\rho+1)} \exp (2 r)^{\rho-\omega}, \quad r \in I$.

Lemma 2 is proven.

6. We fix now the number $\omega$ so that $\rho>\lambda+\omega$.

LEMMA 3. The inequality

$$
\left|R\left(\zeta, w(\zeta), w^{\prime}(\zeta), \ldots, w^{(n)}(\zeta)\right)\right|<r^{s} e^{(3 r)^{\rho-\omega}}
$$

holds for all $\zeta \in H$ with large enough $|\zeta|=r \in I$ (I is defined in Lemma 2) and a certain constant $s$.

Proof. From (1.2) follows

$$
\begin{aligned}
& \left|R\left(\zeta, w(\zeta), w^{\prime}(\zeta), \ldots, w^{(n)}(\zeta)\right)\right| \\
& \quad \leqslant \sum_{i_{0}+i_{1}+\cdots+i_{n}=0}^{m}\left|R_{i_{0} i_{1} \cdots i_{n}}(\zeta)\right|\left|w^{i_{0}}(\zeta)\right|\left|w^{\prime}(\zeta)\right|^{i_{n}} \cdots\left|w^{(n)}(\zeta)\right|^{i_{n}} .
\end{aligned}
$$

According to (5.1) we obtain

$$
\begin{aligned}
& \left|R\left(\zeta, w(\zeta), w^{\prime}(\zeta), \ldots, w^{(n)}(\zeta)\right)\right| \\
& \quad<\sum_{i_{0}+i_{1}+\cdots+i_{n}=0}^{m}\left|R_{i_{0} i_{1} \cdots i_{n}}(\zeta)\right| r^{(\rho+1)\left(i_{0}+i_{1}+2 i_{2}+\cdots+n i_{n}\right)(2 r)} .
\end{aligned}
$$

The functions $R_{i_{0} i_{1}} \cdots i_{n}(z)$ are meromorphic functions of order not higher than $\lambda$ and therefore

$$
\left|R_{i_{0} i_{1}} \cdots i_{n}(\zeta)\right|<e^{(2 r)^{\lambda+e}}, \quad r=|\zeta|>r^{\prime}(\varepsilon),
$$

for $\varepsilon \in H,|\zeta| \in I(\operatorname{see}(5.2))$.

From (6.2) and (6.3) now follows

$$
\begin{array}{r}
\left|R\left(\zeta, w(\zeta), w^{\prime}(\zeta), \ldots, w^{(n)}(\zeta)\right)\right|<A r^{n m(\rho+1)} \exp \left((2 r)^{\rho-\omega}+(2 r)^{\lambda+\varepsilon}\right), \\
\rho-\omega>\lambda+\varepsilon,
\end{array}
$$

where

$$
A=\sum_{i_{0}+i_{1}+\cdots+i_{n}=0}^{m} 1 \text {. }
$$

We see that for $r=|\zeta|>A, r \in I, \zeta \in H$

$$
\left|R\left(\zeta, w(\zeta), w^{\prime}(\zeta), \ldots, w^{(n)}(\zeta)\right)\right|<r^{s} e^{(3 r)^{\rho-\omega}}
$$

with $s=m n(\rho+1)+1$. Thus Lemma 3 is proven.

7. Completion of the proof of the theorem. We know the representation $Q(z, w(z))$ $=f(z) / F(z)$ where $f(z)$ and $F(z)$ are entire functions of order $\langle\lambda$ and $\rho: \rho\rangle \lambda$ respectively. For $\zeta \in H,|\zeta| \in I$ (mes $I=\infty$ ) according to (4.3)

$$
|Q(\zeta, w(\zeta))|<\exp r^{\lambda+\varepsilon} / \exp r^{\rho+\beta(r)}=\exp -r^{\rho+\beta(r)}(1+o(1))
$$

where $\beta(r) \rightarrow_{r \rightarrow \infty} 0$. In view of (6.1) and (7.1) we get for $\zeta \in H,|\zeta| \in I$ 


$$
\begin{aligned}
\mid R\left(\zeta, w(\zeta), w^{\prime}(\zeta),\right. & \left.\ldots, w^{(n)}(\zeta)\right)|| Q(\zeta, w(\zeta)) \mid \\
& <r^{s} \exp (3 r)^{\rho-\omega} \exp -r^{\rho+\beta(r) \cdot(1+o(1))} \\
& =\exp -(1+o(1)) r^{\rho+\beta(r)}
\end{aligned}
$$

(1.1) now gives for the same $\zeta(\zeta \in H,|\zeta| \in I)$

$$
P(\zeta, w(\zeta))=O\left(\exp -(1+o(1)) r^{\rho+\beta(r)}\right) .
$$

The relations

$$
\begin{aligned}
& P(\zeta, w)=O\left(\exp -(1+o(1)) r^{\rho+\beta(r)}\right), \\
& Q(\zeta, w)=O\left(\exp -(1+o(1)) r^{\rho+\beta(r)}\right), \quad \beta(r) \underset{r \rightarrow \infty}{\rightarrow} 0,
\end{aligned}
$$

on the set $\tilde{H}=\{\zeta: \zeta \in H,|\zeta| \in I\}$ define a system of equations, from which $w(\zeta)$ can be found. After eliminating $w$ we obtain

$$
\varphi(\zeta)=O\left(\exp -(1+o(1)) r^{\rho+\beta(r)}\right)
$$

where $\varphi(z)$ is a meromorphic function of order not greater than $\lambda$. Indeed replacing $w$ by $w(\zeta)$, we get according to (1.2)

$$
\begin{aligned}
P_{0}(\zeta) w^{p}+P_{1}(\zeta) w^{p-1}+\cdots+P_{p}(\zeta)= & O\left(\exp -(1+o(1)) r^{\rho+\beta(r)}\right) \\
Q_{0}(\zeta) w^{q}+Q_{1}(\zeta) w^{q-1}+\cdots+Q_{p}(\zeta)= & O\left(\exp -(1+o(1)) r^{\rho+\beta(r)}\right) \\
& (|\zeta|=r, w=w(\zeta), \beta(r) \underset{r \rightarrow \infty}{\rightarrow} 0) .
\end{aligned}
$$

Suppose $p \leqslant q$. We can now eliminate $w^{q}$ by multiplying the first equation of the last system with $Q_{0}(\zeta) w^{q-p}(\zeta)$ and the second with $-P_{0}(\zeta)$ and then adding them. Remembering that $w(\zeta)$ satisfies on $\tilde{H}$ inequality (1.5) (with $\nu=0$ there), we obtain

$$
Q_{0}(\zeta) w^{q-p}(\zeta) O\left(\exp -(1+o(1)) r^{\rho+\beta(r)}\right)=O\left(\exp -(1+o(1)) r^{\rho+\beta(r)}\right)
$$

and

$$
-P_{0}(\zeta) O\left(\exp -(1+o(1)) r^{\rho+\beta(r)}\right)=O\left(\exp -(1+o(1)) r^{\rho+\beta(r)}\right) .
$$

Therefore after the above indicated operations we get

$$
-Q_{1}(\zeta) P_{0}(\zeta) w^{q-1}-\cdots-Q_{q}(\zeta) P_{0}(\zeta)=O\left(\exp -(1+o(1)) r^{\rho+\beta(r)}\right) .
$$

Thus we reduced the degree of one of the equations of (7.4) in respect to $w$ by a unit and the new system from which $w$ has to be eliminated contains the first equation of (7.4) and the last one. In such a way we lower the degrees of $w$ in the equation of (7.4) step by step until $w$ will be completely eliminated and we finally come to

$$
\varphi(\zeta)=O\left(\exp -(1+o(1)) r^{\rho+\beta(r)}\right)
$$

where $\varphi(z)$ is a meromorphic function of order no more than $\lambda$ but which does not vanish identically because $\varphi(z)$ is the resultant of the system $(3.1)$, where $P(z, w)$ and $Q(z, w)$ are mutually prime polynomials in $\mathfrak{R}$. We saw earlier that outside a set $E^{\prime}$ of circles with a finite sum of radii

$$
|\varphi(z)|>e^{-r^{\lambda+e}}
$$

Equality (7.5) now shows that all the solutions of this equation are within $E^{\prime}$. But 
(7.5) is correct for all $\zeta \in \tilde{H}(|\zeta| \in I$, mes $I=\infty)$ so that the set $I$ on the $r$-axis has to be of finite measure: mes $I<\infty$. But mes $I=\infty$. We obtained a contradiction which shows that our assumption about $Q(z, w)$ depending on $w$ is not possible. The theorem is proven.

\section{REFERENCES}

1. B. Ja. Levin, Distribution of zeros of entire functions, GITTL, Moscow, 1956; English transl., Transl. Math. Monographs, vol. 5, Amer. Math. Soc., Providence, R. I., 1964. MR 19, 402; MR 28 \# 217.

2. I. Malmquist, Sur les fonctions à un nombre fini de branches définies par les equations differentielles du premier ordre, Acta Math. 36 (1913), 297-343.

3. Sh. Strelitz, Asymptotic properties of analytic solutions of differential equations, "Mintis", Vilnius, 1972. (Russian)

4. Soc. 65 (1977), 255-261.

DePartment of MAThematics, University of Illinois, Urbana, Illinois 61801

Current address: Department of Mathematics, University of Haifa, Haifa, Israel 